\title{
Intérêt des membranes en polytetrafluoroéthylène de haute densité supportant les expositions en régénération tissulaire guidée: présentation d'un cas de fermeture d'une communication bucco-sinusienne.
}

\author{
Weidmann $\mathrm{P}^{1}$, Tarragano $\mathrm{H}^{1}$, Roche $\mathrm{Y}^{1}$ \\ 1. Pôle d'Odontologie de l'Hôpital Rothschild - Université Paris Diderot - Paris7
}

\section{Introduction}

Une communication bucco-sinusienne (CBS) est une connexion pathologique entre la cavité orale et le sinus maxillaire. Les CBS apparaissent généralement après avulsions de dents antrales ou suite à une complication en chirurgie implantaire. Pour fermer ces CBS, les lambeaux déplacés simples ou associés à une greffe du corps adipeux de la joue sont les techniques les plus communément employées (Visscher SH et al. J Oral Maxillofac Surg, 2010; 68(6):1384-91). La régénération tissulaire guidée (RTG) peut également être employée. Un recouvrement muqueux total de la membrane est alors nécessaire, prévenant une exposition de celle-ci avec risque de réouverture de la CBS. Des membranes nonrésorbables en polytétrafluoroéthylène de haute densité (dPTFE) conçues pour tolérer les expositions au milieu oral sont aujourd'hui disponibles. Cependant, peu d'études cliniques existent dans la littérature scientifique quant à leur utilisation avec exposition délibérée (Carbonell et al. Int J Oral Maxillofac Surg, 2014; 43:75-84). Un cas de fermeture de CBS par mise en place d'une membrane dPTFE exposée est ici présenté.

\section{Matériels et Méthodes}

Le cas d'une femme de 46 ans, sans antécédents médico-chirurgicaux est rapporté. L'examen clinique révélait une suppuration au niveau de couronnes supra-implantaires dans le secteur 1, associée à une douleur spontanée et majorée à la percussion. L'examen tomographique réalisé objectivait la présence d'une lésion radio-claire entourant des implants basaux posés 10 ans auparavant. La lésion osseuse était associée à une CBS de grande étendue (longueur supérieure à $2 \mathrm{~cm}$ ). Cette lésion osseuse et les symptômes cliniques associés ont conduit à la dépose des implants. Après celle-ci, une membrane non-résorbable en dPTFE a été placée au niveau de la CBS et laissée exposée. La stabilisation de la membrane a été obtenue par son enfouissement sous les berges gingivales et par des points de sutures simples.

\section{Résultats}

La membrane dPTFE a été retirée après 8 semaines. La dépose, sans anesthésie, a été réalisée par simple traction de la membrane. Un tissu conjonctif a été retrouvé sous la membrane, faisant obstacle entre la membrane sinusienne et la cavité orale, validant ainsi le succès de la fermeture de la CBS. Par la suite, ce tissu conjonctif a progressivement subi une maturation tissulaire pour adopter un aspect proche de la gencive attachée.

\section{Discussion}

Les résultats rapportés permettent d'élargir l'indication des membranes dPTFE aux fermetures de CBS. L'exposition de ce type de membrane au milieu oral n'a pas perturbé le processus de cicatrisation tissulaire. La capacité des membranes dPTFE à tolérer l'exposition au milieu oral permet d'éviter la traction des tissus mous tout en permettant une fermeture de la CBS.

\section{Conclusion}

La régénération tissulaire avec exposition délibérée d'une membrane dPTFE dans la fermeture de CBS repose sur un protocole simple diminuant la morbidité de l'intervention par rapport à des techniques telles que les lambeaux déplacés avec ou sans greffe du corps adipeux de la joue.

weidmannpierre03@gmail.com

(C) The authors, published by EDP Sciences. This is an Open Access article distributed under the terms of the Creative Commons Attribution License 4.0 (http://creativecommons.org/licenses/by/4.0/). 